OOMMENT. The poor compliance rate which might explain the lack of efficacy of the intermittent diazepam prophylaxis in this study is disappointing and dampens the growing enthusiasm for this form of therapy as a substitute for continuous phenobarbital prophylaxis. The conclusion of Dr. Rosman's controlled study of oral diazepam is eagerly awaited.

In a recent national survey of pediatric neurologists, $22 \%$ recommended the use of intermittent prophylactic therapy with diazepam in an average dose of $0.46 \mathrm{mg} / \mathrm{kg} /$ day; oral diazepam was preferred by $8 \%$ and rectal administration was used by $16 \%$. An alternate drug, lorazepam or clonazepam, was preferred by $2 \%$. (Millichap JG et al. Ann Neurol September 1990; 28:444).

\title{
SHIGELLA-ASSOCIATED RECURRENT SEIZURES
}

The risk of subsequent febrile or nonfebrile seizures following Shigella-associated convulsions was investigated in 55 children in a ten year follow-up study at the Assaf Harofeh Medical Center and the Sackler School of Medicine, Tel Aviv University, Israel. Only two children ( $3 \%$ ) had recurrent episodes of febrile convulsions and none had nonfebrile seizures. The incidence of recurrent seizures in this group of patients was similar to that observed in the general population and significantly different from the average estimated recurrence rate of febrile seizures (33\%). (Lahat E et al. Recurrent seizures in children with Shigella-associated convulsions. Ann Neurol September 1990; 28:393-395).

COMMENT. Among 1292 patients with convulsions associated with Shigellosis in nine publications in the literature, the incidence of seizures varied from $4 \%$ to as high as $45 \%$ with an average of $13 \%$. Among 2241 patients in two studies of Shigella negative diarrheas the incidence of convulsions was only $1.7 \%$. The higher incidence of febrile seizures with shigellosis in comparison with Shigella negative diarrheas was unexplained by neurotoxin formation and could be related to differences in severity of the infections, the height of the fever, and complications of water and electrolyte imbalance. (Millichap JG. Febrile convulsions. Macmillan Company, New York, 1968). The present study suggests that Shigella-associated convulsions are benign and not associated with an increased incidence of either febrile or nonfebrile seizures. An hereditary factor to explain an increased incidence of convulsions with Shigella infections was not supported by this study.

EPILEPSY AND HEMIPARETIC CEREBRAL PALSY

The incidence of epilepsy, the IQ, and laterality of cerebral palsy were investigated in 51 children with hemiparetic CP at the Department of Neurology, Charles University, Katerinska, Praha, Czechoslovakia. Epilepsy had developed in 19; two had partial jacksonian seizures and 17 generalized tonic-clonic seizures. Epileptic paroxysms in the EEG were found in $35(80 \%)$. An IQ of 95 or above was found in 32 and an IQ of 94 or below in 19 . No difference 
in IQ was found between patients with right-sided or left-sided hemiparesis whereas clinical seizures were more cammon in those with right-sided hemiparesis. Clinical epilepsy was related to impaired intellectual performance. Abnormal paroxysmal EEGs were more frequent with left-sided hemiparesis. Among those with clinical epilepsy a lower IQ was found in almost all with left-sided hemiparesis and almost half of those with right-sided hemiparesis. (Sussova $J$ et al. Hemiparetic forms of cerebral palsy in relation to epilepsy and mental retardation. Dev Med Child Neurol Sept 1990; $\underline{32}: 792-795)$.

CONMENT. A lower IQ may be expected in CP patients with clinical epilepsy, especially in those with left-sided hemiparesis. EEG abnormalities alone are not associated with a lower IQ.

\section{BLOOD DISORDERS}

IRON DEFICIENCY ANEMIA AND NEUROLOGIC DEFICITS

A 14 year old black female adolescent with focal neurological abnormalities complicating severe iron deficiency anemia is reported from Duke University Medical Center, Durham, North Carolina. The anemia was caused by bleeding from generalized intestinal polyposis and hereditary hemorrhagic telangiectasia complicated by nasal and gingival bleeding. Neurologic symptams and signs began with occipital headache and neck pain, intermittent diplopia, transient right-sided numbness and weakness, and a brief syncopal episode. On admission the patient was somnolent and the neurological examination revealed bilateral VI nerve palsies, facial palsies, papilledema, and generalized muscle weakness with normal reflexes. After transfusion with packed erythrocytes and treatment with ferrous sulfate orally the facial palsy resolved within 12 hours and the VI nerve palsy and somnolence resolved by the fifth day. A normal hemoglobin was maintained by iron supplementation and the neurologic exam remained normal despite continued gingival and nasal bleeding from telangiectases. (Bruggers CS et al. Reversible focal neurologic deficits in severe iron deficiency anemia. J Pediatr Sept 1990; $117: 430-432$ ).

COMMENT. An iron deficiency anemia is reported in $23 \%$ of cases of breath-holding spells in infants and young children. (Holowach J, Thurston DL. N Eng J Med 1963; 268:21). The neurologic abnormalities with ${ }^{-}$iron deficiency anemia may result from tissue hypoxia, increased capillary permeability, cerebral edema, and abnormal cytochrame enzyme function involved in oxygen metabolism.

VALPROATE-INDUCED CYTOPENIAS

A 16 year old white boy with trisomy 21 and valproic-acid induced erythrocyte aplasia is reported fram the Divisions of Hematology and Oncology, University of Alabama, Birmingham, AL. Suppression of hematopoiesis was demonstrated by in vitro studies of colony-forming- 